\title{
LSST and the Dark Energy Science Collaboration
}

\author{
Jérémy Neveu*† \\ Université Paris-Sud, LAL, UMR 8607, F-91898 Orsay Cedex, France \& CNRS/IN2P3, F-91405 \\ Orsay, France \\ E-mail: jneveulal.in2p3.fr
}

\section{on behalf of the LSST Dark Energy Science Collaboration}

The Large Synoptic Survey Telescope (LSST) is an automated ground-based $8.4 \mathrm{~m}$ optical survey telescope, which should start observations in 2022. The aim of this new instrument is to conduct a ten year wide and deep imaging survey of 18,000 square degrees of the sky in six broad optical bands, with a deep stack reaching magnitude $r \approx 27.5$. The LSST design is driven by four science themes: dark energy and dark matter, galactic structure, transient objects, and the Solar System inventory. After a quick presentation of the project status, I will focus on the LSST cosmological probes. I will highlight the on-going preparation studies about the photometric redshift reconstruction for galaxies and the possibilities in terms of cross-correlations with other cosmological data and surveys.

The European Physical Society Conference on High Energy Physics

5-12 July, 2017

Venice

* Speaker.

${ }^{\dagger}$ on behalf of the LSST/DESC collaboration. 


\section{Introduction}
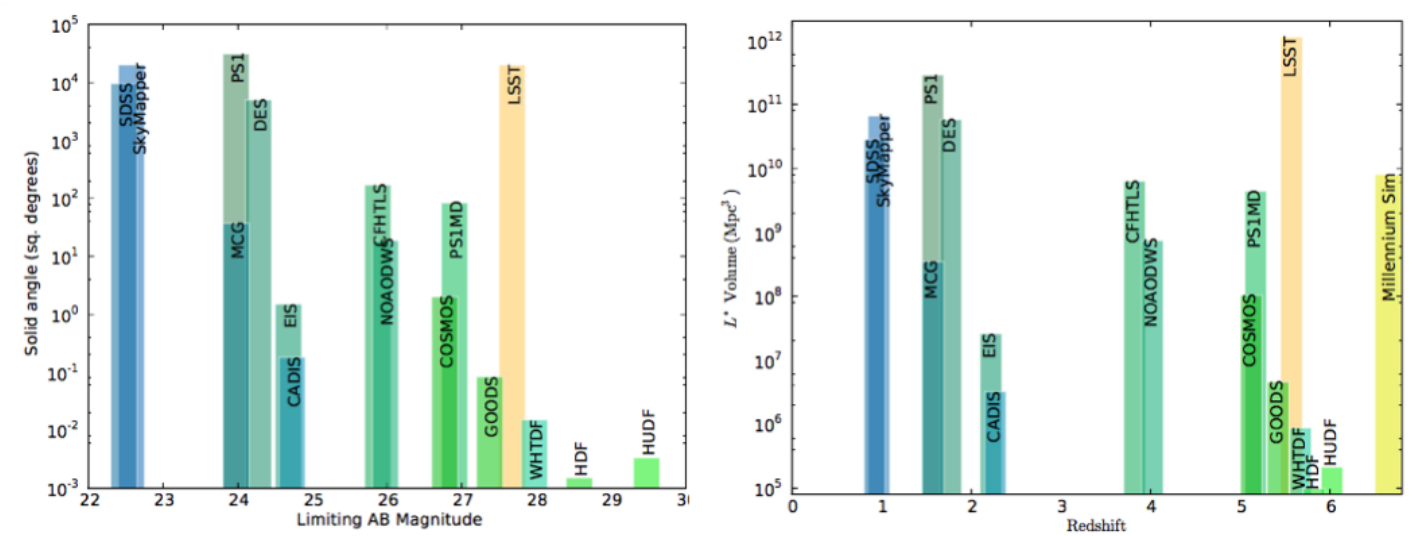

Figure 1: Size of the LSST survey in terms of field size and magnitude, or distances and redshifts, compared with past and future surveys and the Millenium simulation [1].

The Large Synoptic Survey Telescope (LSST) project is an international ground-based telescope project, mostly funded by the US funding agencies. It features a $8.4 \mathrm{~m}$ diameter primary mirror (6.4 m effective) in a compact 3-mirror optical design providing a very wide field of view of $3.5^{\circ}$ diameter. The telescope and its camera are designed to make a survey of the full southern sky $(18,000$ square degrees) every four days, during the 10 years of operation starting in 2022, with 6 broad band-pass filters in the visible spectrum (ugrizy, wavelengths between 350 and 1,200 nm). Each 15-s exposure will reach a magnitude of $r \approx 24$ in the $r$-band ( $5 \sigma$ point source). After around 200 visits on each patch of the sky for each filter, the limiting magnitude in the stacked images magnitude is expected to be $r \approx 27.5$ [1]. LSST will probe the largest volume of Universe ever mapped (Figure 1). The central pieces to make this possible are the very wide 3.2 Gpix camera (with $18916 \mathrm{Mpix}$ CCDs) and the computation chain that will analyze $15 \mathrm{~TB}$ of raw data per night. In 10 years, $50 \mathrm{~PB}$ of raw data are expected, and $10 \mathrm{~PB}$ for the object catalogs.

LSST will cover a large number of astrophysics domains, from Solar System physics (a catalog of $10^{6}$ asteroids is expected), stellar and Milky Way physics ( $10^{10}$ observed stars), and extragalactic physics and cosmology ( $10^{10}$ galaxies and AGNs, and $10^{6}$ type Ia supernovae). These catalogs will bring information about the position and the photometry of the objects, and also their time evolution (proper velocity, luminosity variability, etc.) thanks to the cadence of one visit every four days on average. The width and depth of the survey, its repetition over time, the six colored filters, and the various science goals justify the "synoptic" label in the instrument name.

The LSST telescope and camera have been under construction since 2011, and will be installed on the Cerro Pachón mountain in Chile. The first on-sky calibration images are expected in 2020, and mini-surveys to start a science verification program are scheduled in 2021 . The science program is expected to start in 2022, for 10 years. In parallel, a $1.2 \mathrm{~m}$ diameter auxiliary telescope, CALYPSO, is going to be installed next to the LSST in 2018. Its goal is to contribute to the photometric calibration of the LSST using dedicated spectrographs. 


\section{Cosmology with LSST}

In the following, I focus on the cosmological science to be conducted with the LSST data. The main objectives are the determination of the expansion history of the universe, and the evolution or growth of large-scale structures to constrain the nature of dark energy and dark matter. The dark matter distribution on cosmological scales and the cosmological distance evolution will be revealed thanks to the mapping of weak and strong gravitational lensing effects, the study of the large-scale structures and galaxy clusters, and the detection of type Ia supernovae.

\subsection{The DESC collaboration}

A scientific community interested in dark energy and dark matter studies with the LSST telescope is organized within the Dark Energy Science Collaboration (DESC). The role of the LSST Project team is to provide object catalogs based on the raw images, while the goal of the DESC is to prepare for the higher-level software processing needed to carry out dark energy analysis, including providing feedback to the LSST Project regarding the impact of survey strategy and instrument calibration on dark energy science. In 2017, DESC is composed of 580 Members, with 170 Full Members (who will have privileged access to future data, resources and key publications due to their long-term involvement in DESC). The preparation of the cosmological analyses is detailed in a Science Road Map ${ }^{1}$ and previously in a White Paper [2]. More precisely, the tasks are divided among several Working Groups:

- 7 Analyses Working Groups related to the main cosmological probes: Weak Lensing, Strong Lensing, Large-Scale Structure, Clusters, Supernovae, Theory \& Joint Probes, Photometric Redshifts;

- 3 Software Working Groups: Cosmological Simulations, Survey Simulations and Software \& Computing Infrastructure;

- 2 Technical Working Groups: Sensor Anomalies and Photometric Corrections.

For each Working Group, the tasks must be validated on simulations of increasing complexity until 2019 (3 Data Challenges) or precursor surveys.

In the following I present a selection of science cases considered by the DESC collaboration. The work of the Cluster and Strong Lensing Working Groups is not reported here, but interested readers can find further information in the LSST Science Book [1] and the DESC web site ${ }^{2}$.

\subsection{Photometric redshifts}

As the LSST telescope is not equipped with spectrographs, the galaxy redshifts will be determined using only the photometric measurements through the 6 broad optical filters ugrizy. The photometric redshifts depend critically on the photometric calibration of the instrument and their precision is a key requirement for all the cosmological probes. In particular, the photometric redshift $z$ requirements are (see reference [1] and Figure 2):

\footnotetext{
${ }^{1}$ http://lsst-desc.org/sites/default/files/DESC_SRM_V1_1.pdf

2 http: //lsst-desc.org/
} 

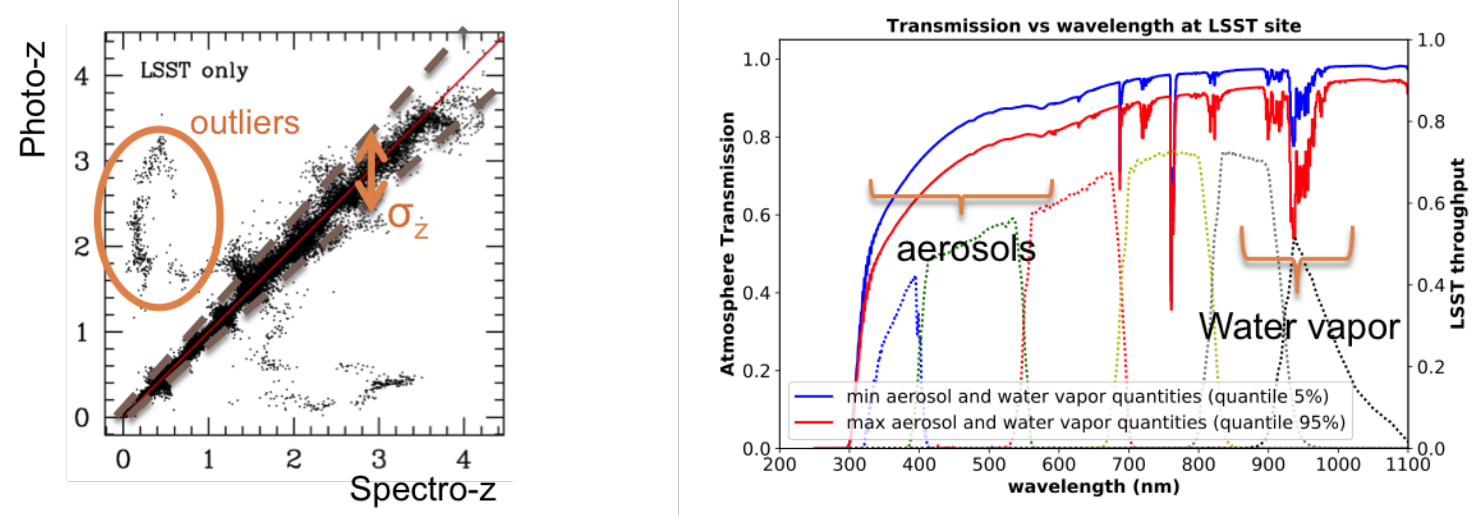

Figure 2: Left: results from a photometric redshift reconstruction on simulation using the LSST optical characteristics, adapted from reference [3]. Right: illustration of the effects of the atmospheric absorption lines and bands on the LSST calibration, for two extreme conditions expected on the LSST site [S. DagoretCampagne courtesy].

- a root-mean-square scatter $\sigma_{z} /(1+z)$ below 0.05 with a goal of 0.02 ;

- a fraction of $3 \sigma_{z}$ outliers below $10 \%$;

- a reconstruction bias $e_{z}=\left(z_{\text {photo }}-z_{\text {spec }}\right) /\left(1+z_{\text {spec }}\right)$ below 0.003 .

These minimal criteria may not be sufficient for a dark energy survey, but a complete and large spectroscopic galaxy sample in the southern hemisphere to train the color-redshift relationship can bring great improvements [4]. Moreover, to improve the calibration of the LSST, the use of the Gaia mission star catalog is considered. Also, to improve the photometric calibration in the $u$ and $y$ bands, the detection of the aerosols and water vapor absorption lines from the LSST auxiliary telescope (Calypso) spectra is being investigated (Figure 2). The goal is to get real-time corrections of the effective filter band-passes due to the variations of the local weather conditions in time and directions.

\subsection{Supernovae}

An approximate total of $10^{6}$ type Ia supernovae ( $\mathrm{SNe} \mathrm{Ia}$ ) is expected to be detected during the 10 years of LSST operations, with $10^{5}$ well enough measured for cosmology, from redshift 0.1 to 1.2. Only a small fraction will benefit from spectroscopic redshifts measured by other telescopes. The large number of events will allow for homogeneity and isotropy tests. For instance several Hubble diagrams can be build in different directions of the sky to test the isotropy of the acceleration of the cosmological expansion, and get clues on the nature of the dark energy. The large sample of supernovae will be useful to conduct detailed investigations on the influence of the host galaxy types on the SNe Ia light curves, and their possible evolution with redshift [1].

To build a proper cosmological sample of SNe Ia, some challenges must be overcome such as the SN classification with photometric information only, and the optimization of the LSST survey cadence to get well-sampled light curves. 


\subsection{Weak lensing}

LSST will probe the growth of structure through the weak lensing signal, either through the measurement of the lensing shear (distorsion of the galaxy shapes), or the lensing magnification, which can be detected by the analysis of the distribution of apparent galaxy magnitudes. The LSST survey will also reveal the lensing of background galaxies by galaxy clusters for a large number of clusters. The analysis of cluster lensing will bring information about the dark matter halos, halo ellipticities, the cluster physics, and the growth of structures. The galaxy shear maps can be analyzed with two-point correlation functions. As approximately ten shear maps are expected at different redshifts between 0 and 3, the auto and cross-correlations of these maps will allow for a redshift tomography of the universe seen in the southern hemisphere. The baseline analysis for the weak lensing in LSST will even correlate the shear maps with the galaxy counting maps to maximize information and enable marginalization over astrophysical systematics. Three-point correlations will be used to bring supplementary information other than the usual matter power spectrum, and control the systematic uncertainties.

For a ground-based telescope, the main systematic uncertainties come from the knowledge of the point-spread function (including spatial variations and wavelength dependence), removal of the impact of the point-spread function from galaxy shapes, and knowledge of photometric redshift errors.

\subsection{Large-scale structure}
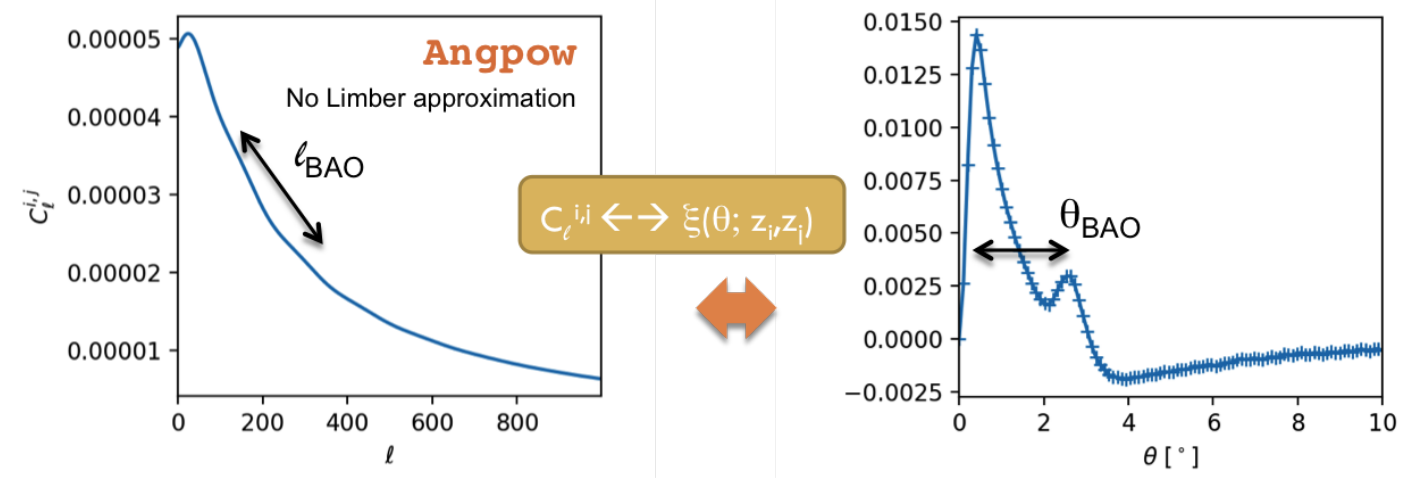

Figure 3: Left: computation of the $C_{\ell}^{i, j}$ spherical harmonics between two redshift bins with the Angpow numerical code [5]. Right: the reciprocal $\xi\left(\theta, Z_{i}, z_{j}\right)$ galaxy correlation function in angular space.

The large-scale structure (LSS) analyses will rely on the large galaxy sample measured by the LSST. The LSS information will be probed directly from the observed variables (angles $\theta$ and redshifts $z$ ), using galaxy-galaxy angular separation correlation functions $\xi(\theta)$ or spherical harmonics $C_{\ell}$. These observables are more suitable for galaxy distributions spread on half a sphere, and with rather large uncertainties on their redshifts, compared with spectroscopic redshifts. It is also more convenient than the usual two-point correlation functions in real space $\xi(r)$ or the Fourier matter power spectrum $P(k)$ as data analysis does not have to assume any fiducial cosmological model to convert angles and redshifts into distances, but works directly in the observable space. As for the shear maps, galaxies can be split into different redshift bins: auto and cross-correlations between 
them can be conducted to extract information on the cosmological parameters. The Angpow and Angcorr numerical codes were developed to compute precisely and quickly the theoretical auto and cross-correlations $\xi\left(\theta ; z_{1}, z_{2}\right)$ and $C_{\ell}\left(z_{1}, z_{2}\right)$ between two bins at redshift $z_{1}$ and $z_{2}$ (Figure 3). These codes compute exact correlation functions without the Limber approximation, which is often used to speed up the computation but fails at large-scales and for cross-correlations [5, 6].

The pipelines of the LSS analyses are under construction and the impact of the photometric redshift misreconstructions, in particular those of the outliers, is under investigation. Other systematic uncertainties to handle are the selection function in magnitude and the Milky Way dust absorption.

\subsection{Theoretical computations}

One major accomplishment of the Theory \& Joint Probe Working Group so far is the building of a numerical code, the Core Cosmological Library (CCL), to share a common framework to compute the cosmological observables (cosmological distances and linear perturbations) among the DESC collaboration. The goal of CCL is to provide a well-documented code, whose accuracy has been controlled and validated, to all the cosmological Working Groups so that DESC cosmological analyses are consistent. It lies on hardcoded cosmological observables but also on the interface with other trusted numerical codes such as CLASS [7] and Angpow [5]. CCL has been publicly released and a related publication is expected at the end of 2017.

\section{Conclusions}

The LSST is a multi-probe program for cosmology: it will conduct cosmological analyses using most of the available cosmological probes. It will be possible to use cross-correlations between the different probes (such as the shear and the galaxy distribution) in order to constrain the cosmological parameters and control the systematic uncertainties (galaxy bias, photometric redshifts, weak lensing intrinsic alignment, etc.) [8]. Combining all the probes, the expected uncertainty is below the percent level for a constant equation of state parameter of dark energy $w$, or $10 \%$ for a dynamical $w_{0}-w_{a}$ parameterization of the dark energy equation of state [2].

The cosmological parameters can be further constrained exploiting the complementarities between LSST and the upcoming instruments, such as the Euclid and WFIRST space missions [3], or the SKA radio telescope. All these experiments could benefit from each other, for instance to enhance photometric redshifts and to control systematics. Also the synergies between LSST and the LIGO and Virgo gravitational wave detectors, and the X-ray observatories could bring optical counterparts useful for the transient search and the birth of the gravitational wave astronomy.

\section{References}

[1] LSST Science Collaborations, LSST Science Book, 2009, arXiv:0912.0201.

[2] LSST Dark Energy Science Collaboration, 2012, arXiv:1211.0310.

[3] Jain B., Spergel D., Bean R., et al., 2015, arXiv:1501.07897. 
[4] Newman J., Abate A., Abdalla F., et al., 2015, Astroparticle Physics, 63, 81-100, arXiv:1309.5384.

[5] Campagne J., Neveu J. \& Plaszczynski S., 2017, A\&A, 602, A72, arXiv:1701.03592.

[6] Campagne J., Plaszczynski S. \& Neveu J., 2017, ApJ, 845, 28, arXiv:1703.02818.

[7] Blas D., Lesgourgues J. \& Tram T., 2011, Cosmol.Astropart.Phys., 034.

[8] Rhodes J., Allen S., Benson B.A., et al., 2013, arXiv:1309.5388. 